\title{
Impact of revised antiretroviral treatment on the immunological, virological and clinical parameters among people infected with HIV
}

\author{
Jaya Lalwani ${ }^{1, *}$, Camilla Rodrigues ${ }^{2}$, Rajeev Soma ${ }^{3}$ \\ ${ }^{\mathbf{1}}$ Associate Professor, Dept. of Microbiology, Gandhi Medical College, Bhopal, Madhya Pradesh, ${ }^{2}$ Consultant Microbiologist, \\ ${ }^{3}$ Consultant Physician, ${ }^{2}$ Dept. of Microbiologist, ${ }^{3}$ Dept. of Physician, P. D. Hinduja National Hospital and Medical Research \\ Centre, Maharashtra, India
}

*Corresponding Author:

Email: drjaya_is@yahoo.co.in

Received: $1^{\text {st }}$ May, 2018

Accepted: $1^{\text {st }}$ June, 2018

\begin{abstract}
Introduction: In resource-limited countries like India, where resistance testing is often not available, an accurate treatment history can guide doctors in quantifying suboptimal drug exposure and thus anticipate drug resistance.

Materials and Methods: In the present longitudinal study, a total of 75 patients failing antiretroviral therapy; the effective drug exposure was calculated after taking into account the adherence, appropriateness of prescriptions, and pharmacokinetic interactions. Treatment was modified and patients were followed up.

Results: Of the total 75 patients included in the study; 69 (92.0\%), 63 (84.0\%) and $42(56.0 \%)$ patients had virological, immunological and clinical failure respectively. Suspected reasons for antiretroviral drug failure were non-adherence in 48 $(64.0 \%)$, improper prescriptions in $12(16.0 \%)$ and drug interactions in $12(16.0 \%)$. We observed that following treatment change, CD4 count increased by a mean of $127 \mathrm{cells} / \mu \mathrm{l}$ and $33(44.0 \%)$ patients had fully suppressed viral loads.

Conclusion: Our results show that empirical treatment changes based on a comprehensive drug history, followed by good adherence lead to good treatment outcomes in patients showing one or other evidence of drug resistance.
\end{abstract}

Keywords: HIV, AIDS, Drug-resistance, Antiretroviral.

\section{Introduction}

Antiretroviral therapy (ART) has significantly reduced mortality and morbidity in individuals with human immunodeficiency virus infection. ${ }^{1}$ At the same time, ART has succeeded in improving the quality of life of people living with HIV/AIDS. ${ }^{2}$ However, it is noticed that very often patients switch to alternate drug combinations due to drug toxicity, intolerable adverse effects, inconvenience or costs, but also due to worsening clinical outcomes. ${ }^{3}$ Among people living with HIV/AIDS (PLHA) reappearance of HIV RNA in plasma may or may not be always associated with drug resistance mutations (DRM). DRM is frequently due to poor adherence but is also due to a high genetic barrier to resistance for some drugs. ${ }^{4}$

To date, only a few observational studies have been conducted to assess the virological response to ARV and have reported conflicting results on the consequences for disease progression by the various patterns of drug resistance and treatment failure..$^{5}$ Also, it is well known that the viral load (VL) levels at treatment initiation play a determinative role in the first-line treatment response and the development of DRM. ${ }^{6} \mathrm{We}$, therefore, studied the outcome of empirical treatment change in patients failing the first-line antiretroviral treatment. The present study was conducted with the objective to determine the factors related to suboptimal drug exposure and outcome of renewed empirical treatment.

\section{Materials and Methods}

Study Design: This was a longitudinal study.

Source of Study Participants: all the patients registered for receiving ARV treatment at P. D. Hinduja medical centre were enrolled in the present study.

Sample Size: all patients fulfilling the inclusion criteria during the period of study. Following this norm, we enrolled a total of 75 patients failing antiretroviral therapy.

Case Definition: Definition of failure of therapy was based on a combination of clinical, immunological, and virological parameters. ${ }^{7,8}$ A comprehensive and detailed history of clinical progression of the disease and the treatments received till date were recorded. Selfreported adherence to the antiretroviral therapy was recorded. Improper prescriptions were noted and interacting drugs were identified from available prescription slips. Patients were counselled regarding the importance of adherence to antiretroviral drugs prior to changing treatment.

Follow-up: The duration of follow-up varied; the period of following ranged from minimum three months to a maximum of one year. We attempted to quantify effects on drug exposure by considering the kinetic interactions. For example, rifampicin is known to reduce nevirapine levels by as much as $58 \% .^{9-11}$ Therefore; it was considered that the patient had $42 \%$ drug exposure to nevirapine while on rifampicin.9-11 Also, inappropriate prescription involving monotherapy or dual therapy was noted. If only 2 drugs of 3 were prescribed, drug exposure was considered as $67 \%$ for 
that period of time. The effective drug exposure over the entire treatment duration was calculated after taking into consideration all these factors. We considered slabs of $0-15 \%, 16-53 \%, 54-73 \%, 74-94 \%$ and $95-100 \%$ for adherence on the basis of previous studies. ${ }^{9-12}$ Since the relationship between drug exposure and resistance is bell-shaped, it was anticipated that low $(<50 \%)$ or high level $(>90 \%)$ of drug exposure would be associated with low levels of resistance. ${ }^{9-12}$ Such patients would be expected to do well with the original regimen at least in short term. On the other hand, drug exposures between $50-90 \%$ would be associated with a high likelihood of resistance. $^{9-12}$ Lamivudine and NNRTIs have a low genetic barrier to resistance and there is complete crossresistance between the NNRTIs. All these drug interactions and other pharmacokinetic factors were taken into accounts in anticipating resistance and changing treatment empirically.

\section{Results}

All study participants were assessed for clinical improvement by weight gain, immunological and virological parameters. Our study included 75 patients failing antiretroviral therapy. Of these 75 patients, each of the $24(32.0 \%)$ had exclusive immunological and virological failures respectively. Whereas, 33 (44.0\%) patients had all three types viz. clinical, virological and immunological failures (Table 1).

Table 1: Distribution of study participants by the type of treatment regimen failure $(n=75)$

\begin{tabular}{|l|c|c|}
\hline S. No. & Type of failure & No. of patients n (\%) \\
\hline 1 & Only Clinical & 00 \\
\hline 2 & Only Immunological & $03(4.0)$ \\
\hline 3 & Only Virological & $06(8.0)$ \\
\hline 4 & Clinical + Immunological & $03(4.0)$ \\
\hline 5 & Clinical + Virological & $06(8.0)$ \\
\hline 6 & Immunological + Virological & $24(32.0)$ \\
\hline 7 & Clinical + Immunological + & $33(44.0)$ \\
\hline
\end{tabular}

The possible reason for failure in study participants were non-adherence $(48,64.0 \%)$, incorrect prescriptions $(12,16.0 \%)$ and drug interactions (12, $16.0 \%$ ) (Table 2). Genotypic resistance testing could be done in $15(20.0 \%)$ of these 75 patients and the results correlated $100 \%$ with the anticipated resistance to various drugs.

Table 2: Distribution of study participants by self-reported reason for failure $(\mathbf{n}=75)$

\begin{tabular}{|l|c|c|}
\hline S. No. & Reason & $\mathbf{n}(\mathbf{\%})$ \\
\hline 1 & Non-adherence & $48(64.0)$ \\
\hline 2 & Incorrect/inappropriate prescriptions & $12(16.0)$ \\
\hline 3 & Pharmacokinetic drug interactions & $12(16.0)$ \\
\hline 4 & Possible drug resistance (Long-term HAART) & $01(1.3)$ \\
\hline
\end{tabular}

Following a change in the antiretroviral treatment, the weight of these patients increased by a mean of 2.14 kilograms. The CD4 count after modification of treatment was $>500$ cells $/ \mu \mathrm{l}$ in 4 patients, between 200 500 cells $/ \mu \mathrm{l}$ in 11 patients and $<200$ cells $/ \mu \mathrm{l}$ in 10 patients. There was an increase in CD4 count from a mean value of 151 cells $/ \mu$ before altering treatment to
278 cells $/ \mu \mathrm{l}$ after modifying treatment. As can be seen from table 3 , after a change of treatment, $33(44.0 \%)$ out of 75 patients had fully suppressed viral load of $<50$ copies. Viral load was between 54-10000 in 18 (24.0\%) patients, $10000-100000$ in 15 patients, while 9 (12.0\%) patients had a viral load of $>100000$ copies $/ \mu l$.

Table 3: Distribution of study participants by a change in viral load $(n=75)$

\begin{tabular}{|l|c|c|c|}
\hline S. No. & Viral load & \multicolumn{2}{|c|}{ No. of patients } \\
\cline { 3 - 4 } & & Before treatment n (\%) & After treatment n (\%) \\
\hline 1 & $<50$ & $06(8.0)$ & $33(44.0)$ \\
\hline 2 & $50-10,000$ & $15(20.0)$ & $18(24.0)$ \\
\hline 3 & $10,001-100,000$ & $18(24.0)$ & $5(6.7)$ \\
\hline 4 & $>100,000$ & $36(48.0)$ & $09(12.0)$ \\
\hline
\end{tabular}




\section{Discussion}

Of the 75 study participants $69(92.0 \%)$ had virological and 63(84.0\%) had immunological failure whereas; clinical failure was evident in $42(56.0 \%)$ of them. These figures underscore the fact that laboratory monitoring of CD4 counts and viral loads is of utmost importance in detecting early treatment failure in patients with HIV. Before modifying treatment, there were only 6 patients who had a viral load of $<50$ but had an immunological failure, clinical progression and were clearly on suboptimal treatment. It is possible that presence of $\mathrm{M} 184 \mathrm{~V}$ mutation might have prevented the rise in viral load, although resistance must be present in these patients. ${ }^{13,14}$ Although, it was found that among about $64 \%$ of patients the possible causes of treatment failure were non-adherence to therapy, $32.0 \%$ patients had inappropriate prescriptions and drug interactions as a cause of failure. These circumstances may be peculiar to our setting where the physician and patient education programs are suboptimal. ${ }^{15}$ Of the total 75 study participants included in the study, only one patient failed despite appropriate treatment and full adherence possibly as an inevitable consequence of long-term HAART. ${ }^{16}$ Current guidelines recommend resistance testing to optimize drug selection after treatment failure. ${ }^{17,18}$ However, resistance tests require a resistant viral population of more than $10-20 \%$ to detect resistance. They may not predict hyper-susceptibility or efficacy of combinations and boosting. ${ }^{19}$ Finally, the resistance tests are expensive and are not generally available. ${ }^{20,21}$ Thus in our study, the resistance testing could be performed only in $15(20.0 \%)$ patients. It was observed that the results correlated well with the drugs to which the resistance was anticipated.

\section{Conclusion}

Our results show that empirical treatment changes based on a comprehensive drug history, followed by good adherence lead to good treatment outcomes in patients showing one or other evidence of drug resistance. Using immunological criteria to predict which patient has not achieved virological suppression results in significant misclassification of therapeutic responses. There is an urgent need for the availability of viral load testing in initiation as well as monitoring of ART. Also, the development of standardized and universally accepted definitions of virological failure is necessary to allow meaningful therapeutic interventions.

\section{Limitations}

In our opinion following were the limitations of the present study. Firstly, the adherence as defined in this study was self-reported by the patients. Although the optimal way to assess adherence to antiretroviral therapy is not known, self-reported adherence appears to be the most feasible method. ${ }^{9-12}$ In our study, CD4 cell count and viral loads were not always done at the same laboratory. The consequence of poor adherence might be different when the viral load was expected to be high than when it was low. This might have important implications for anticipating resistance in that one might give a different weight to early nonadherence as compared to late non-adherence. This factor could not be taken into consideration in this study. Despite these limitations, our results show that in patients failing antiretroviral therapy empiric treatment change followed by good adherence and drug exposure leads to good clinical, immunologic and virological outcome.

\section{Conflict of Interest: None}

\section{Source of Funding: None}

\section{References}

1. Palella FJ Jr., Delaney KM, Moorman AC, Loveless MO, Fuhrer J, Satten GA, et al. Declining morbidity and mortality among patients with advanced human immunodeficiency virus infection. HIV Outpatient Study Investigators. N Engl J Med. 1998;338(13):853-60.

2. Lepri AC, Miller V, Phillips AN, Rabenau H, Sabin CA, Staszewski $\mathrm{S}$. The virological response to highly active antiretroviral therapy over the first 24 weeks of therapy according to the pre-therapy viral load and the weeks 4-8 viral load. AIDS. 2001;15(1):47-54.

3. Lucas GM, Chaisson RE, Moore RD. Highly active antiretroviral therapy in a large urban clinic: risk factors for virologic failure and adverse drug reactions. Ann Intern Med. 1999;131:81-7. Epub 07/27.

4. Vo TT, Ledergerber B, Keiser O, Hirschel B, Furrer H, Battegay M, et al. Durability and outcome of initial antiretroviral treatments received during 2000-2005 by patients in the Swiss HIV Cohort Study. The Journal of infectious diseases. 2008;197(12):1685-94.

5. Gupta R, Hill A, Sawyer AW, Pillay D. Emergence of drug resistance in HIV type 1-infected patients after receipt of first-line highly active antiretroviral therapy: a systematic review of clinical trials. Clinical infectious diseases: an official publication of the Infectious Diseases Society of America. 2008;47(5):712-22.

6. Wainberg MA, Zaharatos GJ, Brenner BG. Development of antiretroviral drug resistance. $N$ Engl J Med. 2011;365(7):637-46.

7. Mellors JW, Munoz A, Giorgi JV, Margolick JB, Tassoni CJ, Gupta P, et al. Plasma viral load and CD4+ lymphocytes as prognostic markers of HIV-1 infection. Ann Intern Med. 1997;126(12):946-54.

8. Metzner KJ, Giulieri SG, Knoepfel SA, Rauch P, Burgisser P, Yerly S, et al. Minority quasispecies of drugresistant HIV-1 that lead to early therapy failure in treatment-naive and -adherent patients. Clinical infectious diseases: an official publication of the Infectious Diseases Society of America. 2009;48(2):239-47.

9. Lohse N, Jorgensen LB, Kronborg G, Moller A, Kvinesdal B, Sorensen HT, et al. Genotypic drug resistance and long-term mortality in patients with tripleclass antiretroviral drug failure. Antiviral therapy. 2007;12(6):909-17.

10. Lucas GM. Antiretroviral adherence, drug resistance, viral fitness and HIV disease progression: a tangled web is woven. J Antimicrob Chemother. 2005;55(4):413-6. 
11. Lucas GM, Gallant JE, Moore RD. Relationship between drug resistance and HIV-1 disease progression or death in patients undergoing resistance testing. AIDS. 2004;18(11):1539-48.

12. Cozzi-Lepri A, Phillips AN, Clotet B, Mocroft A, Ruiz L, Kirk O, et al. Detection of HIV drug resistance during antiretroviral treatment and clinical progression in a large European cohort study. AIDS. 2008;22(16):2187-98.

13. Zaccarelli M, Tozzi V, Lorenzini P, Trotta MP, Forbici F, Visco-Comandini U, et al. Multiple drug class-wide resistance associated with poorer survival after treatment failure in a cohort of HIV-infected patients. AIDS. 2005;19(10):1081-9.

14. Santoro MM, Armenia D, Alteri C, Flandre P, Calcagno A, Santoro M, et al. Impact of pre-therapy viral load on virological response to modern first-line HAART. Antivir Ther. 2013;18(7):867-76.

15. Neogi U., Häggblom A., Santacatterina M., Bratt G., Gisslén M., Albert J., \& Sonnerborg A. (2014). Temporal trends in the Swedish HIV-1 epidemic: increase in non-B subtypes and recombinant forms over three decades. PloS one, 9(6), e99390.

16. Stanford University HIV Drug Resistance Database. [cited 20131 th of May]. Available from: 2013.

17. Johnson VA1, Brun-Vezinet F, Clotet B, Gunthard HF, Kuritzkes DR, Pillay D, Schapiro JM, Richman DD. Update of the Drug Resistance Mutations in HIV-1. Top HIV Med, 2008;16:138-45.
18. Liu TF, Shafer RW(2006). Web Resources for HIV type 1 Genotypic-Resistance Test Interpretation. Clin Infect Dis. 42(11):1608-18.

19. Charpentier C, Dwyer DE, Mammano F, Lecossier D, Clavel F, Hance AJ. Role of Minority Populations of Human Immunodeficiency Virus Type 1 in the Evolution of Viral Resistance to Protease Inhibitors. Journal of Virology. 2004;78(8):4234-4247.

20. Ramadhani HO, Bartlett JA, Thielman NM, Pence BW, Kimani SM, Maro VP, et al. Association of first-line and second-line antiretroviral therapy adherence. Open forum infectious diseases. 2014;1(2):ofu079.

21. Napravnik S, Eron JJ, Sterling TR, Juday T, Uy J, Moore RD. Outcomes of Second Combination Antiretroviral Therapy Regimens Among HIV-Infected Persons in Clinical Care: A Multicenter Cohort Study. AIDS research and human retroviruses. 2013;29(3):574-80.

How to cite this article: Lalwani J, Rodrigues C, Soma R. Impact of revised antiretroviral treatment on the immunological, virological and clinical parameters among people infected with HIV. Indian J Microbiol Res. 2018;5(3):374377 . 